\title{
Standing open magnetic resonance imaging improves detection and staging of pelvic organ prolapse
}

\author{
Lynn Stothers, MD 1,2,3; Jennifer A. Locke, MD, PhD'; Marwa Abdulaziz, PhD'; Darren Lazare, $M D^{3}$; \\ Alex Kavanagh, $M D^{1,2}$; Andrew Macnab, $M D^{1,4}$
}

'Department of Urologic Sciences, University of British Columbia, Vancouver, BC, Canada; ${ }^{2}$ International Collaboration on Repair Discoveries (ICORD); ${ }^{3}$ Department of Obstetrics and Gynecology, University of British Columbia, Vancouver, BC, Canada; ${ }^{4}$ Stellenbosch Institute for Advanced Study, Wallenberg Research Centre at Stellenbosch University, South Africa

\begin{abstract}
Cite as: Stothers $L$, Locke JA, Abdulaziz $M$, et al. Standing open magnetic resonance imaging improves detection and staging of pelvic organ prolapse. Can Urol Assoc J 2022;16(1):E20-4. http://dx.doi.org/10.5489/cuaj.7244
\end{abstract}

Published online August 26, 2021

Appendix available at cuaj.ca

\section{Abstract}

Introduction: The role of imaging in pelvic organ prolapse (POP) assessment is unclear. Open magnetic resonance imaging (MRI) systems have a configuration that allows for imaging women with POP in different positions. Herein, we use a 0.5 Tesla open MRI to obtain supine, seated, and standing images. We then compare these images to evaluate the impact of posture on detection and staging of POP.

Methods: Women presenting with symptoms of POP at a tertiary care university hospital were asked to participate in this prospective cohort study. Symptom scores, POP-Q staging and three-position MRI imaging of the pelvis data were collected. The pubococcygeal line $(\mathrm{PCL})$ was used to quantify within-patient changes in pelvic organ position as defined by: no displacement, $<1 \mathrm{~cm}$ inferior to the PCL, mild $(1-3 \mathrm{~cm})$, moderate $(3.1-6 \mathrm{~cm})$, and severe $(>6 \mathrm{~cm})$ in the axial and sagittal T2-weighted images. Statistical analysis was completed (T-test; $\mathrm{p}<0.05$ significant).

Results: A total of 42 women, age range $40-78$ years, participated. There was a significant difference in the mean values associated with anterior prolapse in the supine $(0.7 \pm 1.8)$, seated $(2.4 \pm 3.4)$, and upright $(4.2 \pm 1.6)$ positions $(p=0.015)$. There was a significant difference in the mean values associated with apical prolapse in the supine $(0.5 \pm 1.5)$, seated $(1.5 \pm 1.4)$, and upright (2.1 \pm 1.5$)$ positions $(p=0.036)$.

Conclusions: Our findings suggest that POP is more readily detected and upstaged with standing MRI images as compared to supine and seated positions. The developed two-minute standing MRI protocol may enable clinicians to better assess the extent of POP.

\section{Introduction}

The International Continence Society guideline for management of pelvic organ prolapse (POP) states that, "Imaging may assist the clinical assessment of POP or intercurrent pelvic floor diagnoses," but also emphasizes that it is entirely optional. ${ }^{1}$ There is recognition that gravity and posture affect the interplay of the pelvic support structures/organs and hence influence the detection and staging of POP. ${ }^{2-5}$ Currently, clinicians rely on the POP-Q score for staging and therapeutic decision-making for POP; ${ }^{6}$ however, physical examination in the upright position or when the patient performs a Valsalva maneuver during the POP-Q assessment are known to have inherent limitations. ${ }^{6-8}$ Magnetic resonance imaging (MRI) has been proposed to be a useful method for diagnosing and staging POP; ${ }^{1}$ however, conventional scanners require the patient to be in a supine position, thus limiting visualization of the effects of posture and gravity.

Re-operation rate in POP is "unacceptably high and likely represents an underestimate of the true rate." ${ }^{\prime 9}$ DeLancey proposes that approximately $30 \%$ of the operations performed are re-operations, and states, "The high prevalence of this problem indicates the need for treatment improvement, which will only be possible if research clarifies causative biomechanical mechanisms and scientifically valid studies discover why operations fail." 10 Suboptimal staging of POP with the POP-Q and conventional MRI assessments, due to the inability to capture the full influence of posture and gravity, may be a reason for such a high re-operation rate.

Hence, we propose imaging in the upright position, particularly in those women with symptoms of POP but negative POP-Q results. Open MRI systems allows patients to be imaged supine, seated, standing, and fully weight bearing. In 1996, the concept of MRI imaging in the upright position was introduced by Fielding et al. ${ }^{11}$ Evaluating five volunteers with the open MRI, most of the pelvic floor structures were stable, with the exception of the posterior urethrovesical angle, which was increased in the sitting position as com- 
pared to the standing position. Apart from a study by Grob et $\mathrm{al}_{,}{ }^{2}$ which investigated the effect of upright scanning on staging with patients straining, the potential for standing MRI to add to the evaluation of POP was not explored further until a study by Friedman et al. ${ }^{12}$ Friedman et al compared MRI images of 11 women in the supine and standing positions and found differences in bladder position in patients with POP between the two positions $(p=0.03) .{ }^{12}$ This was not observed in controls. As these studies included very few patients, standing MRI is not part of the imaging recommendations from international bodies. ${ }^{1,13}$

While it is assumed that an accurately performed POP-Q physical exam equates to an accurate diagnosis, we know that surgical failure rates are a concern. The objective of this study was to use a 0.5 Tesla open MRI scanner to obtain supine, seated, and standing images, and compare them to evaluate the impact of posture on detection and staging of POP in women. We propose a role for open MRI evaluation in women with symptoms of POP and a negative POP-Q assessment.

\section{Methods}

\section{Study population}

Following ethical approval (UBC Ethics H14-03507), we recruited women aged 18 years and older with symptomatic POP from female pelvic medicine subspecialty practices of urologists/urogynecologists at multiple tertiary care, university-based medical practices. All subjects were surgically naive for prolapse surgery. Initial evaluation and staging of patients included a complete history, incorporating validated symptom questionnaires of the Urogenital Distress Inventory (UDI-6), ${ }^{14,15}$ the Colorectal-Anal Distress Inventory (CRADI 8), ${ }^{16}$ and the Pelvic Organ Prolapse Distress Inventory (POPDI-6). ${ }^{17}$ Physical examination included height, weight, urinalysis, postvoid residual, and POP-Q staging.

\section{Imaging}

All subjects were screened using the MRI safety checklist. Open MRI of the pelvis was performed using a 0.5 Tesla system (Paramed Medical Systems, Italy) located at the University of British Columbia Centre for Hip Health. Subjects emptied their bladder prior to entering the scanner. To avoid any anatomical distortion, neither contrast agents nor a surface body coil was used.

The dynamic sequence of imaging acquisition was supine, followed by seated views, followed by transition to the standing position. Parameters used to obtain axial and sagittal views of the full pelvis in the supine and seated positions were: TR 470 TE 10; echo train length, 32; bandwidth, 32 $\mathrm{kHz}$; excitation, one; matrix size, $256 \times 160$; field of view,
100; section axial T2-weighted images with thickness $5 \mathrm{~mm}$ and slice gap $1 \mathrm{~mm}$. The protocol used a T2-weighted singleshot fast spin-echo (SSFSE) sequence (TR: 1300 ms; TE: 60 ms; slice thickness: $6 \mathrm{~mm}$; field of view 32-36 cm; matrix: 256×160; 1 excitation and half-Fourier acquisition).

Subjects were then transitioned to the standing position, and to assist with balance and stability, a support bar was provided for patients to hold on to. The upright scan was limited to a $10 \mathrm{~cm}$ field of view centered on the pubis to limit the time patients had to stand still. The standing image acquisition time was two minutes, in recognition that some patients find standing immobile for a long time difficult or uncomfortable. In addition, to address the possibility of subjects feeling faint when upright, all patients wore leg deep venous thrombosis external pneumatic compression devices.

\section{Pelvic reference lines}

To quantify the degree of descent of the pelvic organs and to compare the differences between standing, seated, and supine imagines in $\mathrm{cm}$, pelvic reference lines were used. The pubococcygeal line (PCL) was drawn as a line extending from the most inferior portion of the symphysis pubis to the tangent of the last coccygeal joint. ${ }^{18}$ Differences between the supine, seated, and upright distance of the bladder neck, cervix, and pouch of Douglas from the PCL were measured in $\mathrm{cm}$. Pelvic organ position was defined as: no displacement or $<1 \mathrm{~cm}$ inferior to the PCL, mild $(1-3 \mathrm{~cm})$, moderate $(3.1-6 \mathrm{~cm})$, and severe $(>6 \mathrm{~cm})$.

\section{Statistical analysis}

Demographic and clinical questionnaire data were reported as mean with standard deviation. Displacement related to the PCL was compared between supine, seated, and upright position using $\mathrm{IBM}^{\circ}$ SPSS statistical software for analysis, with t-test $p<0.05$ indicating significance.

\section{Results}

\section{Patient demographics}

Forty-two women with symptoms of POP participated. The mean age was 65 years (range 40-78). Eight percent had undergone forceps delivery. Thirteen were premenopausal and 29 post-menopausal. Parity, smoking history, and validated symptom scores are shown in Table 1 and in the Appendix (the latter available at cuaj.ca). Mean body mass index (BMI) was $33.5 \mathrm{~kg} / \mathrm{m}$ (range 20-38). Mean POP-Q stage supine was 2.0 (range $0-3.5$ ) and POP-Q stage upright was 2.5 (range 0-3.5). 


\begin{tabular}{|c|c|c|c|c|}
\hline & \multicolumn{4}{|c|}{ Symptomatic subjects POP $(\mathrm{N}=42)$} \\
\hline \multirow[t]{2}{*}{ Parity } & 0 & 1 & 2 & 3 \\
\hline & 1 & 15 & 20 & 6 \\
\hline \multirow[t]{2}{*}{ Smoking history } & Never & & & Remote \\
\hline & 35 & & & 7 \\
\hline
\end{tabular}

MRI: magnetic resonance imaging; POP: pelvic organ prolapse.

Supine, seated, and standing images of good diagnostic quality were obtained in all 42 subjects. A composite image comparing supine, seated, and standing position in the same subject is shown in Fig. 1. Specifically, all subjects were able to complete the two-minute standing sequence without evidence of motion artefact. Results comparing mild, moderate, and severe prolapse based on the PCL line are shown in Table 2. There was a significant difference in the mean values associated with anterior prolapse in the supine $(0.7 \pm 1.8)$, seated $(2.4 \pm 3.4)$, and upright $(4.2 \pm 1.6)$ positions $(p=0.015)$. There was a significant difference in the mean values associated with apical prolapse in the supine $(0.5 \pm 1.5)$, seated $(1.5 \pm 1.4)$, and upright $(2.1 \pm 1.5)$ positions ( $p=0.036)$.

Five of the 42 women had a negative POP-Q assessment for prolapse in both supine and upright but on the POPDI 6 , answered affirmatively to question 2 relating to having a bulge that they could see or feel. In these five subjects, prolapse was identified when imaged in the standing position (Fig. 2). In brief, anterior prolapse observed in the upright scan was not seen in the supine scan.

\section{Discussion}

Overall, standing MRI imaging identified a greater degree of prolapse in symptomatic women with POP compared to supine imaging. These results suggest that open MRI should have a role in evaluating women with symptoms of POP, especially if they are found to have a negative POP-Q assessment. We also note that our protocol, which includes a short scan time and central field of view using the 0.5 Tesla MRI scanner, provides images of high diagnostic quality and can be applicable in subjects with a broad range of BMIs. The open MRI scanner has been primarily used in the context of orthopedic care for the evaluation of weight-bearing effects and the impact of gravity on joints and ligaments. ${ }^{19,20}$ This application of open MRI to better define and stage POP builds on our initial pilot studies applying the technology in urology. ${ }^{12,21}$

In the 42 women studied, no downstaging occurred when comparing upright to supine images. Importantly, in the five subjects with symptoms of protrusion but no detectable prolapse on POP-Q exam, prolapse was identified in the standing open MRI images.
The relevance of what we report relates to several clinical scenarios. Women who present with a clear history suggestive of prolapse can have negative findings on a clinical exam. The literature discusses reasons for this, including supine positioning, patient inability to Valsalva effectively, and limited ability to distinguish all organ positions during standing exam; ${ }^{2}$ however, in the context of imaging, none of the European Society of Urogenital Radiology Pelvic Floor Working Group or the European Society of Gastrointestinal and Abdominal Radiology or the International Urogynecology Assocation recommendations include standing position for standard MRI imaging in pelvic floor dysfunction. ${ }^{1,13}$

In addition, the choice of surgical procedure is primarily based on the stage of prolapse defined clinically. The greater detail of pelvic anatomy and enhanced definition of organ relationships provided by standing imaging may allow for greater refinement in surgical correction of POP, as the clinician is able to chose a procedure that better matches the anatomical defect(s). We propose that the additional information standing images provide will help reduce the rate of postoperative failure. For example, a patient who presents with only anterior prolapse on POP-Q exam but is discovered, upon standing MRI, to have apical prolapse, may benefit from repair of both anterior and apical prolapse in terms of preventing postoperative failure.

Although we found a statistical difference in stage between seated and supine images, we suggest that there is no clinical benefit to seated imaging in women who are able to stand. The rationale for studying subjects when seated was

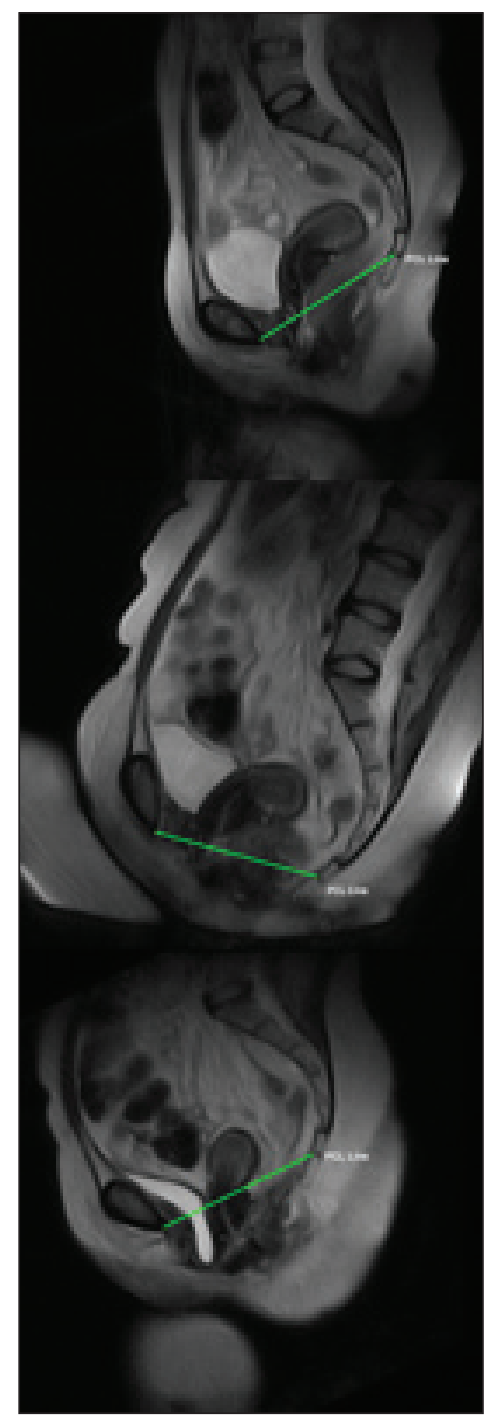

Fig. 1. A composite image of (A) supine, $(\boldsymbol{B})$ seated, and (C) upright scans in a 65-year-old G2P2 post-menopausal female with prolapse staged using the pubococcygeal line. The standing image demonstrates the presence of significant prolapse upstaging pelvic organ prolapse (POP) in this subject. 


\begin{tabular}{|c|c|c|c|c|}
\hline & & Mild & Moderate & Severe \\
\hline \multirow[t]{2}{*}{ Supine } & Anterior prolapse & $6 \%$ & $1 \%$ & $1 \%$ \\
\hline & Apical prolapse & $22 \%$ & $1 \%$ & $1 \%$ \\
\hline \multirow[t]{2}{*}{ Upright } & Anterior prolapse & $36 \%$ & $42 \%$ & $12 \%$ \\
\hline & Apical prolapse & $36 \%$ & $12 \%$ & $1 \%$ \\
\hline
\end{tabular}

to aid in the evaluation of women who cannot weight bear, such as those with neurological conditions. This is illustrated in Fig. 1, where a prolapse identified in the standing position is not demonstrated when seated. lacobellis et al also found differences in organ position between the supine and seated position. ${ }^{22}$ In our study, some subjects' upstaging was evident when seated, but the full extent was not demonstrated until standing. A further consideration is that women who have severe prolapse detected in the supine position may compress the prolapse when seated, and this can result in artificial downstaging of the extent of POP.

We suggest that the protocol described for supine and standing image acquisition can be used clinically; the combination of using a 0.5 Tesla open magnet and the ability to image patients when standing upright allowed the full effect of posture and gravity to be evident and provided diagnostic-quality images. Also, because the standing image segment of the protocol involves a short image acquisition time (two minutes), as a limited central field of view is scanned, it is possible to scan almost anyone who can stand. Keeping the duration of the standing sequence short also reduces potential motion artifact.

Importantly, this protocol was designed to be non-invasive, free of radiation, and practical clinically, with short duration and without coils or contrast. Because this patient group frequently has lower urinary tract symptoms, the potential exists for stress or urgency incontinence during the study. Hence, patients voided prior to being scanned and none were incontinent. We suggest that aiming to standardize bladder volume for this investigation without catheterization is not practical and would convert the protocol into an invasive study. Furthermore, many women with POP have incomplete bladder emptying as part of their condition.

Grob et al reported the effect of upright scanning on staging with patients straining. ${ }^{2}$ They found the distance from the bladder neck, cervix, and pouch of Douglas to the PCL increased significantly from the supine straining position relative to an upright rest and straining position; however, this study involved a small presurgical cohort of 15 subjects who had stage 2 or higher POP. The authors state that image quality obtained was not optimal; their technique required the use of a coil, a lower Tesla magnet, and inclined rather than fully vertical imaging. In addition, their protocol was restricted, as they could not image subjects with U.S. jean size 14 or greater, and the National Health and Nutrition Examination Survey indicates that $66.9 \%$ of women in the U.S. are overweight or obese (defined as BMI 25-29.9 for overweight and 30 or more for obese). ${ }^{23}$

Our ability to quantify upstaging through upright imaging builds on the discipline of using pelvic reference lines. ${ }^{13,18}$ A technical point when considering the benefits of standing images is the current practice of viewing supine images in a vertical orientation and publishing illustrations of them in the same manner. We suggest that for clarity, with the availability of upright images, supine images should be shown horizontally and standing images vertically.

We recognize the limitations in what we report. In this study, patients voided prior to starting the MRI and filled spontaneously throughout the study. The International Consultation on Incontinence recommendation is that patients should have an empty bladder during physical examination to avoid a filled bladder causing an obstruction on descent during the Valsalva maneuver, leading to a missed POP diagnosis. ${ }^{24}$ Patients did not strain, cough, or Valsalva; this was for practical reasons, to limit the duration of the protocol, limit motion artifact, and ensure image quality. Reporting of posterior prolapse was not included; our protocol was designed to be non-invasive and hence avoid contrast. Comparison with women without POP was not made; control data have been published previously. ${ }^{12}$ Patients were screened for contraindications to safe conduct of MRI; not all patients with POP will be eligible. This technique does require access to an open MRI facility. These are increasing in number and may be a viable option in the future. All participants were sufficiently able-bodied to be able to stand; in practice, some patients will be too compromised in this regard to be able to complete the standing segment of the protocol or will be wheelchair-bound. Due to the cost of each MRI study, we were limited by funding

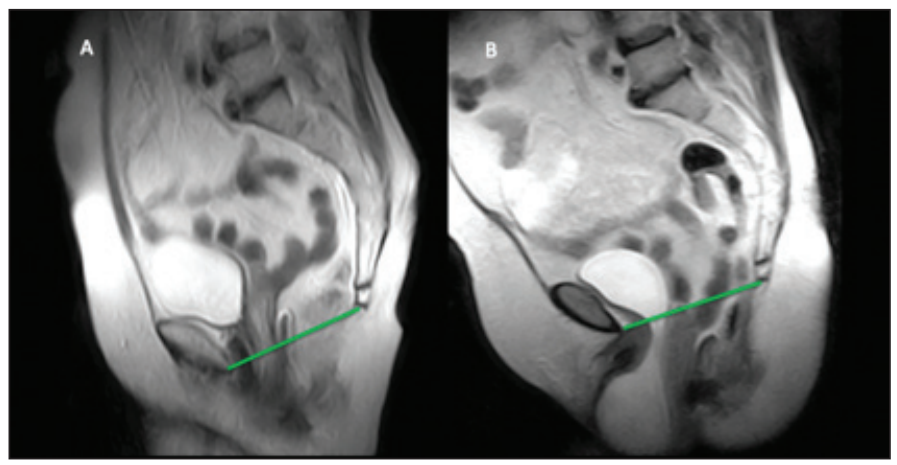

Fig. 2. A sample image demonstrating the extent to which gravity dependent pelvic organ prolapse (POP) can be upstaged by comparing standing to supine images. A 60-year-old G1P1 female who presented with increasing urinary incontinence and a feeling of vaginal heaviness when ambulant. On pelvic exam supine and inclined, at rest and with Valsalva, revealed no organ descent. Evaluation using upright open imaging shows $(\boldsymbol{A})$ supine and $(\boldsymbol{B})$ standing imaging confirming evidence of prolapse with the effects of posture and gravity. This shows also shows the adequacy of the abbreviated twominute scan for diagnostic purposes. 
restraints to recruiting only 42 patients in this study; it may be of benefit to evaluate more women with symptoms on POP in future studies using this technology.

Future directions could include:

- The investigation of pre and postoperative patients to understand the anatomical changes achieved surgically and how these are affected by upright posture. This could potentially impact surgical technique for primary and secondary procedures.

- A randomized clinical trial to compare the surgical outcomes in those women who have negative POP-Q assessments who undergo MRI evaluation with a positive finding and those who do not to determine the preoperative role of standing MRI in surgical planning/counselling.

- Exploration of other pelvic reference lines to define the impact of upright posture on quantification of rectal prolapse. In complex cases, 3D image reconstruction from upright images can be applied where the protocol allows for high-quality images to be obtained. ${ }^{21}$

- Biomechanical models of pelvic organ relationships; ${ }^{10}$ these are currently done using supine images, and are one area to explore in future research using standing imaging.

- Exploration of how the POP-Q stage could be complimented by the addition of standing imaging data. The image quality that can be obtained using this protocol may have applicability to understanding changes to the female urethra based on posture and gravity.

\section{Conclusions}

Standing images obtained using open MRI technology allow the influence of posture and gravity on POP to be identified. Comparison of standing to supine images indicates statistically significant upstaging in both anterior prolapse and apical prolapse. The developed two-minute standing MRI protocol may enable clinicians to better assess the extent of POP, especially in those with a negative POP-Q assessment.

Competing interests: The authors do not report any competing personal or financial interests related to this work.

Acknowledgements/funding: The authors would like to thank the Vancouver Coastal Research Institute Translational research award program for funding support.

This paper has been peer-reviewed.

\section{References}

1. Haylen BT, Maher CF, Barber MD, et al. An International Urogynecological Association (IUGA)/International Continence Society (ICS) joint report on the terminology for female pelvic organ prolapse (POP). Neurourol Urodyn 2016;35:137-68. hitps://doi.org/10.1002/nau.22922
2. Grob ATM, Olde Heuvel J, Futterer JJ, et al. Underestimation of pelvic organ prolapse in the supine straining position, based on magnetic resonance imaging findings. Int Urogynecol J 2019;30:1939-44. hitps://doi.org/10.1007/s00192-018-03862-0

3. Bø K. Pelvic floor muscle strength and response to pelvic floor muscle training for stress urinary incontinence. Neurourol Urodyn 2003;22:654-8. https://doi.org/10.1002/nau.10153

4. Barber MD, Lambers $A$, Visco AG, et al. Effect of patient position on clinical evaluation of pelvic organ prolapse. Obstet Gynecol 2000;96:18-22. https://doi.org/10.1016/S0029-7844(00)00859-0

5. Hendrix SL, Clark A, Nygaard I, et al. Pelvic organ prolapse in the Women's Health Initiative: Gravity and gravidity. Am J Obstet Gynecol 2002;186:1160-6. https://doi.org/10.1067/mob.2002.123819=

6. Bump RC. The POP-Q system: Two decades of progress and debate. Int Urogynecol J 2014;25:441-3. https://doi.org/10.1007/s00192-013-2262-0

7. Bump RC, Mattiasson A, Bø K, et al. The standardization of terminology of female pelvic organ prolapse and pelvic floor dysfunction. Am J Obstet Gynecol 1996;175:10-7. https://doi.org/10.1016/S00029378(96)70243-0

8. Riss P, Dwyer PL. The POP-Q classification system: Looking back and looking forward. Int Urogynecol J 2014;25:439-40. https://doi.org/10.1007/s00192-013-2311-8

9. Denman MA, Gregory WT, Boyles SH, et al. Reoperation 10 years after surgically managed pelvic organ prolapse and urinary incontinence. Am J Obstet Gynecol 2008;198:555.e l-5. https://doi.org/10.1016/i.ajog.2008.01.051

10. DeLancey J0. The hidden epidemic of pelvic floor dysfunction: Achievable goals for improved prevention and treatment. Am J Obstet Gynecol 2005;192:1488-95. https://doi.org/10.1016/i.ajog.2005.02.028

11. Fielding JR, Versi E, Mulkern RV, et al. MR imaging of the female pelvic floor in the supine and upright positions. J Magn Reson Imaging 1996;6:961-3. https://doi.org/10.1002/imri.1880060622

12. Friedman B, Stothers L, Lazare D, et al. Positional pelvic organ prolapse (POP) evaluation using open, weight-bearing magnetic resonance imaging (MRI). Can Urol Assoc J 2015;9:197-200. https://doi.org/10.5489/cuai.2767

13. El Sayed RF, Alt CD, Maccioni F, et al. Magnetic resonance imaging of pelvic floor dysfunction - joint recommendations of the ESUR and ESGAR Pelvic Floor Working Group. Eur Radiol 2017;27:2067-85. https://doi.org/10.1007/s00330-016-4471-7

14. Shumaker SA, Wyman JF, Uebersax JS, et al. Health-related quality of life measures for women with urinary incontinence: The incontinence impact questionnaire and the urogenital distress inventory. Continence Program in Women (CPW) Research Group. Qual Life Res 1994;3:291-306. https://doi.org/10.1007/ BF00451721

15. Cam C, Sakalli M, Ay P, et al. Validation of the short forms of the incontinence impact questionnaire (IIQ-7) and the urogenital distress inventory (UDI-6) in a Turkish population. Neurourol Urodyn 2007;26:129-33. https://doi.org/10.1002/nau.20292

16. Barber MD, Kuchibhatla MN, Pieper CF, et al. Psychometric evaluation of 2 comprehensive condition-specific quality of life instruments for women with pelvic floor disorders. Am I Obstet Gynecol 2001;185:1388-95. https://doi.org/10.1067/mob.2001.118659

17. Barber MD, Walters MD, Bump RC. Short forms of two condition-specific quality-of-life questionnaires for women with pelvic floor disorders (PFDI-20 and PFIQ-7). Am J Obstet Gynecol 2005;193:103-13. https://doi.org/10.1016/i.ajog.2004.12.025

18. Betschart C, Chen L, Ashton-Miller JA, et al. On pelvic reference lines and the MR evaluation of genital prolapse: a proposal for standardization using the pelvic inclination correction system. Int Urogynecol J 2013;24:1421-8. htrps://doi.org/10.1007/s00192-013-2100-4

19. Yamamura M, Miki H, Nakamura N, et al. Open-configuration MRI study of femoro-acetabular impingement. J Orthop Res 2007;25:1582-8. https://doi.org/10.1002/jor.20448

20. Johal P, Williams A, Wragg P, et al. Tibio-femoral movement in the living knee. A study of weight bearing and non-weight bearing knee kinematics using 'interventional' MRI. J Biomech 2005;38:269-76. https://doi.org/10.1016/i.jbiomech.2004.02.008

21. Abdulaziz M, Deegan EG, Kavanagh A, e al. Advances in basic science methodologies for clinical diagnosis in female stress urinary incontinence. Can Urol Assoc J 2017;11:S117-20. hitps://doi.org/10.5489/ cuaj.4583

22. lacobellis F, Brillantino A, Renzi A, et al. MR imaging in diagnosis of pelvic floor descent: Supine vs. sitting position. Gastroenterol Res Pract 2016;2016:6594152. https://doi.org/10.1155/2016/6594152

23. NIH Overweight \& Obesity Statistics. 2021. Available at: https://www.niddk.nih.gov/health-information/ health-statistics/overweight-obesity. Accessed April 9, 2021.

24. Abrams P, Andersson KE, Apostolidis A, et al. 6th International Consultation on Incontinence. Recommendations of the International Scientific Committee: Evaluation and treatment of urinary incontinence, pelvic organ prolapse and fecal incontinence. Neurourol Urodyn 2018;37:2271-2. https://doi.org/10.1002/nau.23551

Correspondence: Dr. Lynn Stothers, Department of Urologic Sciences, University of British Columbia, Vancouver, BC, Canada; lynns@mail.ubc.ca 